\section{LAS UNIDADES REGIONALES DE INVESTIGACIÓN EN SALUD, PERÚ 2009}

\section{[REGIONAL UNITS IN HEALTH RESEARCH, PERU 2009]}

\author{
L. Patricia Yamaguchi ${ }^{1, a}$, Martin Yagui ${ }^{1, b}$, \\ Percy Mayta-Tristán ${ }^{2, c}$
}

Sr. Editor. La generación de nuevos conocimientos que respondan a las necesidades de salud de la población es la base para tener mejores sistemas de salud (1), para ello se requiere de la existencia de sistemas nacionales de investigación en salud (SNIS), que tienen prioridades, políticas, estrategias y financiamiento para generar y aplicar el conocimiento que se traduzca en el bienestar de su población ${ }^{(2)}$.

Diversos países en desarrollo han logrado establecer SNIS, teniendo como resultado políticas de salud basadas en evidencias ${ }^{(3)}$. Para que funcione un SNIS no solo debe haber una institución rectora a nivel nacional (que en el Perú es el Instituto Nacional de Salud [INS]), sino que deben existir interlocutores a nivel regional o local que gestionen la investigación que requieren en sus localidades ${ }^{(4)}$. Desde el año 2007, se ha iniciado un proceso de descentralización de las funciones de salud a cada región del Perú, entre ellas la función de gestión de investigación a cargo de las Direcciones Regionales de Salud (DIRESA).

En nuestro país, el SNIS se encuentra en fase de construcción, uno de los primeros pasos para llegar a su organización incluye identificar quienes se responsabilizarán de la gestión de la investigación en cada región, por ello el objetivo del estudio fue conocer las características de las unidades de investigación (UI) de las DIRESA del Perú.

Se realizó una encuesta entre los meses de julio y agosto de 2009, incluyéndose a todas las DIRESA del Perú (28), a las que se envío un cuestionario que incluyó preguntas sobre la estructura de la organización de las UI, las características del personal que la conforma y la gestión de la investigación que realizan. Se identificó

Oficina General de Investigación y Transferencia Tecnológica, Instituto Nacional de Salud. Lima, Perú.

2 Oficina General de Información y Sistemas, Instituto Nacional de Salud. Lima, Perú.

a Enfermera epidemióloga; ${ }^{b}$ Médico epidemiólogo. ${ }^{\circ}$ Médico salubrista.

$$
\text { Recibido: 25-08-10 Aprobado: 01-09-10 }
$$

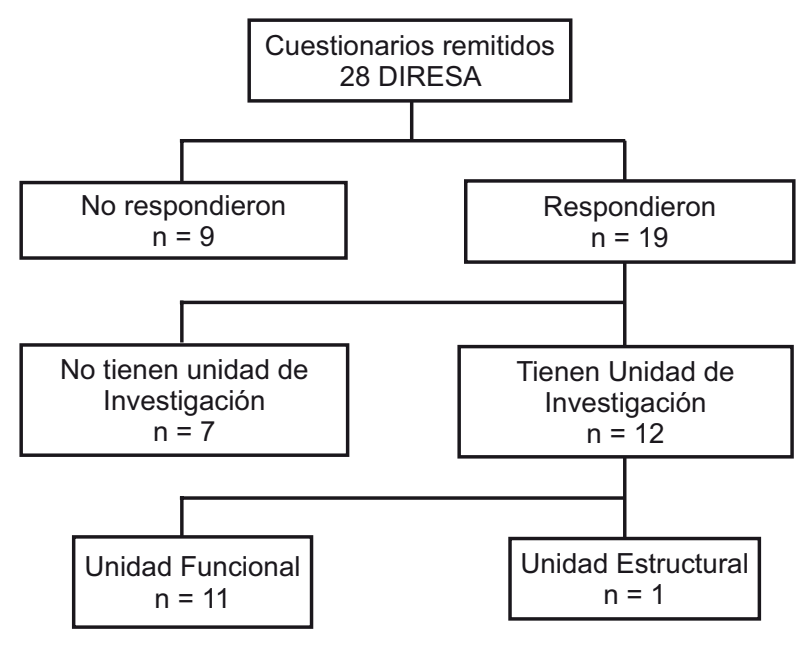

Figura 1. Respuesta al cuestionario según la presencia de unidades de investigación en las direcciones de salud del Perú, 2009.

a cada uno de los encargados de las unidades de investigación, luego se realizó una búsqueda de sus publicaciones en Medline, SciELO, LILACS y Scholar Google.

De 28 cuestionarios remitidos, se recibieron 19 respuestas, 7/19 manifestaron que no tenían UI, de las 12 DIRESA que tenían UI, siete tenían una resolución directoral que reconocía su existencia, pero solo una era estructural (Figura 1).

Por otro lado, 9/12 habían desarrollado algún tipo de normatividad sobre investigación, pero la mayoría relacionadas con la elaboración de protocolos de investigación. En cuanto a la infraestructura, 11/12 disponen de computadoras, pero solo cinco tienen acceso a Internet y seis a teléfono.

De las 19 que respondieron, 15 manifestaron que realizaban funciones de investigación, cuatro de ellas dependían de la Oficina de Epidemiología, nueve de la Oficina de Recursos Humanos y dos de la Dirección General, en todas ellas se ubicó al menos a un responsable de las funciones de investigación.

Los responsables de las funciones de investigación (15) tienen en promedio cuatro años de trabajo en la institución, diez de ellos son nombrados. Según profesión, diez son profesionales de la salud (tres médicos) y los demás de otras áreas, 5/15 no refieren haber tenido algún tipo de capacitación previa en investigación, solo uno de ellos publicó en una revista científica en español. Únicamente 2/15 refieren que se dedican en forma exclusiva a cumplir funciones de investigación, el resto comparte estas actividades con otras funciones relacionadas con la dependencia donde laboran. 
Trece DIRESA refieren que realizan la revisión de protocolos de investigación y 11 manifiestan aprobarlos; aunque solo seis de ellas cuentan con un Comité de Investigación y dos tienen un Comité Institucional de Ética en Investigación para la aprobación de sus protocolos. Diez tienen un registro de investigaciones, seis tienen incluidas las actividades de investigación dentro del plan operativo institucional y únicamente tres refieren que tienen algún tipo de supervisión sobre las investigaciones que aprueban.

Para estimular las actividades de investigación en sus jurisdicciones, nueve DIRESA han desarrollado convenios con universidades, ocho cuentan con un registro de investigadores locales, aunque no son disponibles desde sus páginas web, doce refieren haber identificado sus prioridades de investigación.

Estos resultados muestran que existen, aunque en forma no estructurada, algunas unidades regionales de investigación, lo que serviría de base para cumplir con las funciones de gestión de la investigación transferidas. Es conocido que la gestión de la investigación requiere de un equipo con personal preparado y a dedicación exclusiva ${ }^{(5,6)}$, esta falencia se refleja en la evaluación que realizó el Ministerio de Salud sobre el cumplimiento de la función de gestión de la investigación a nivel regional, donde la media alcanzada a nivel nacional fue del $7 \%$, siendo esta la función transferida en salud con menor avance de todas. Posterior a ello, se realizó el proceso de identificación de prioridades de investigación en salud a nivel regional y nacional liderado por el INS, actualmente todas las regiones tienen prioridades identificadas.

Los responsables de estas unidades deben recibir mayor capacitación sobre el tema, idealmente deben ser parte del equipo personas que conozcan el proceso de investigación, que hayan investigado y publicado.

Otro aspecto básico para el avance y solidificación de la investigación a nivel regional es que existan Comités de Ética en Investigación (CEI); sin embargo solo 2/28 cuentan con uno, lo que limita las posibilidades de desarrollar investigación y es un aspecto a trabajar a futuro.

Este estudio presenta la situación basal de las UI a nivel regional antes de una política de desarrollo descentralizado de la investigación en el Perú a cargo del INS. Estos resultados denotan la necesidad de institucionalizar la investigación en salud a nivel regional a través de la creación o fortalecimiento de las unidades de investigación de manera orgánica en las unidades ejecutoras del sector salud (DISA/DIRESA/ hospitales y otras afines), de tal manera que permitan el planeamiento, organización y sostenibilidad de los procesos y procedimientos de investigación en salud así como incrementar su calidad.

En conclusión, en el Perú dos tercios de las regiones poseen unidades de investigación funcionales, con escaso personal y múltiples actividades que limitan el desarrollo de la función de gestión de la investigación a nivel regional, se requiere el apoyo para su fortalecimiento tanto a nivel regional (DIRESA) como del INS.

\section{Fuente de Financiamiento} Instituto Nacional de Salud

\section{Conflictos de Interés}

LPY y MY trabajan en el desarrollo de las unidades de investigación a nivel regional y nacional.

\section{REFERENCIAS BIBLIOGRÁFICAS}

1. Pang T, Sadana R, Hanney S, Bhutta ZA, Hyder AA, Simon J. Knowledge for better health-a conceptual framework and foundation for health research systems. Bull World Health Organ. 2003; 81(11): 815-20.

2. Hanney SR, González Block MA. Building health research systems to achieve better health. Health Res Policy Syst. 2006; 4:10

3. World Health Organization. National Health Research Systems. A report of an international workshop. Geneva: WHO; 2002.

4. Pedrera Carbonell V, Gil Guillén V, Orozco Beltran D. Unidades de investigación y docencia de apoyo a la gestión en atención primaria. Aten Primaria. 2003; 32(6): 361-65.

5. Lansang MA, Dennis R. Building capacity in health research in the developing world. Bull World Health Organ. 2004; 82(10): 764-70.

6. Da Silva T, Szklo F, Barata RB, de Noronha JC. Evaluation of the Brazilian Health Research System: some characteristics of the researchers and the scientific production. Elect J Commun Inf Innov Health. 2007; 1(1): 9-18.

Correspondencia: L. Patricia Yamaguchi Dirección: Cápac Yupanqui 1400, Lima 11. Teléfono: (511) 6176200 anexo 2268

Correo electrónico: lyamaguchi@ins.gob.pe 